\title{
DIMENSIONAMENTO DE ESTACIONAMENTO PARA VEÍCULOS DE CARGA E DESCARGA
}

\author{
Luzenira Alves Brasileiro ${ }^{1}$
}

Camila Ferreira de Ascenção

Thales Alexandre Rosin ${ }^{3}$

\begin{abstract}
RESUMO
O estacionamento para veículo de carga e descarga é o tempo necessário para carregamento ou descarregamento de carga, na forma disciplinada pelo órgão de trânsito competente. Muitos impactos negativos são gerados com as operações não planejadas de carga e descarga em centro comercial, destacando-se as restrições de tráfego; aumento do risco sobre os demais veículos e pedestres; conflitos entre o transporte de passageiros e carga; e obstruções ao trânsito. Sem o planejamento do transporte de carga em zona comercial, também há redução da velocidade do trânsito em geral e formação de congestionamentos; aumentando o tempo de viagem e o custo do próprio transporte de carga, aumentando conseqüentemente 0 custo final dos produtos. Portanto, o processo de distribuição de carga deve ser planejado, de modo a não gerar o caos urbano. $O$ objetivo deste trabalho é dimensionar dois estacionamentos para veículos de carga e descarga, um em cada sentido da via principal (na Zona Norte), visando suprir a demanda de lojas no local. O método utilizado para o dimensionamento é a técnica de Pesquisa Operacional denominada Teoria de Filas. As áreas de estacionamento do caso em estudo necessitam de 8 vagas no segmento Norte-Sul e de 6 vagas no segmento Sul-Norte. Conclui-se que é de grande importância uma ação conjunta entre o planejamento urbano e a operação dos veículos de carga e descarga, pois a existência de uma área específica para estas operações aumenta a fluidez e diminui os conflitos de trânsito.
\end{abstract}

PALAVRAS-CHAVE: Área de estacionamento. Veículos. Operação de carga e descarga.

\section{AREAS OF PARKING FOR LOADING AND UNLOADING VEHICLES}

\footnotetext{
${ }^{1}$ Universidade Estadual Paulista (UNESP) Campus Ilha Solteira, luzenira@dec.feis.unesp.br

2Universidade Estadual Paulista (UNESP) Campus Ilha Solteira, cfdeas@gmail.com

${ }^{3}$ Universidade Estadual Paulista (UNESP) Campus Ilha Solteira, thales.rosin@gmail.com
} 


\begin{abstract}
Parking for loading and unloading vehicles should ensure the time required to load or unload, established and regulated by the institution or entity with constituency on the way done. Many negative impacts are generated by unplanned operations for loading and unloading, especially traffic restrictions; increased risk to all other vehicles and pedestrians; disputes between passengers and freight; traffic and obstructions. Therefore, the load distribution process must be planned to not generate the urban chaos. The lack of planning in freight traffic in urban center slows traffic in general and generates traffic jam, increasing travel time and the cost of freight transport itself, increasing consequently the final cost of the products. The purpose of this paper is to scale two parking areas for loading and unloading vehicles, one in each direction of the main street. The method used for scaling is the technique of Operational Research calledQueueTheory. The parking area of the case study requires 8 spaces in the North-South direction and 6 spaces in the South-North direction. It can be concluded that it is of great importance a joint action between urban planning and the operation of vehicles for loading and unloading, since the determination of a specific area for these operations increases the fluidity and reduces traffic conflicts.
\end{abstract}

KEY-WORDS: Parking. Loading. Unloading.

\title{
DIMENSIONAMIENTO DE ESTACIONAMIENTO PARA VEHÍCULOS DE CARGA Y DESCARGA
}

\section{RESUMEN}

El aparcamiento para vehículos de carga y descarga debe asegurar el tiemponecesario para que se hagan la carga o descarga, establecida y regulada por la institución o entidad con circunscripción sobre la vía. Muchos impactos negativos son generados por las operaciones no planeadas de carga y descarga, resaltándose las restricciones de tráfico; aumento del riesgo en todos los demás vehículos y peatones; conflictos entre pasajeros y carga; y obstrucciones al tráfico. Por lo tanto, el proceso de distribución de carga debe ser planificado a fin de no generar el caos urbano. La falta de planificación del transporte de mercancías en el centro comercial lentifica el tráfico en general y genera embotellamiento, aumentando el tiempo de viaje y el costo del transporte de mercancías, con el consiguiente aumento de coste final de los productos. El objeto de este trabajo es hacer el dimensionamiento de dosestacionamientos para vehículos de carga y descarga, uno en cada sentido de circulación. El método utilizado para el dimensionamiento es la técnica de Investigación de Operaciones llamada de Teoría de Colas. La zona de aparcamiento del estudio requiere 8 plazas en el sentido Norte-Sur y 6 plazas en el sentido Sur-Norte. Se puede concluir que es de gran importancia una acción conjunta de la planificación urbana y de la operación de los vehículos de carga y descarga, ya que la determinación de un área específica para estas operaciones aumenta la fluidez y reduce los conflictos del tráfico.

PALABRAS-CLAVE: Estacionamiento. Carga. Descarga. 


\section{INTRODUÇÃO}

As questões relacionadas ao estacionamento de veículos são de interesse estratégico para o trânsito e para a ordenação do espaço público. Assim, a regulamentação de área para veículos de carga e descarga deve ser compatível com o plano diretor do desenvolvimento urbano da cidade, e orientar de maneira efetiva a entidade de fiscalização de trânsito.

Quando há a falta de área específica para estacionamento de carga e descarga em centro comercial são gerados problema em três segmentos: (1) a área urbana sofre um desequilíbrio funcional que gera prejuízos econômicos; (2) o sistema de carga sofre um aumento nos custos de transporte, que incide em um acréscimo no custo final do produto; (3) a mobilidade urbana é afetada pelo conflito entre o transporte de carga e passageiros, gerando acidentes e congestionamentos de trânsito. Portanto, as áreas para estacionamento de carga e descarga devem ser planejadas de forma a não ocorrer o caos urbano, que também provoca deterioração na qualidade de vida da sociedade.

As operações de carga e descarga são fundamentais para o funcionamento do sistema urbano e para o crescimento econômico das cidades, mas devem ser realizadas em áreas específicas para manter a harmonia do trânsito e o desenvolvimento local. As vagas de estacionamento para veículos de carga e descarga devem ser dimensionadas em função do tamanho do veículo (pequeno, médio e grande), e os espaços de manobra e acesso devem ser dimensionados em função do ângulo formado pelo comprimento da vaga e a faixa de acesso.

\section{OBJETIVOS}

O objetivo deste trabalho é analisar como ocorrem as operações de carga e descarga ao longo da avenida principal de llha Solteira - SP (Av. Brasil) e dimensionar dois estacionamentos próprios para tais veículos, um em casa sentindo, de modo a suprir a demanda das lojas existentes no local. 


\section{MATERIAL E MÉTODO}

A simulação é uma das técnicas utilizadas para o dimensionamento de estacionamentos. A simulação é uma ferramenta de planejamento disponibilizada para Pesquisa Operacional (PO), permitindo a geração de cenários, a partir dos quais se pode orientar o processo de tomada de decisão, permitir análises e avaliações de desempenho e propor soluções para a melhoria de sistemas.

Os modelos de simulação têm grande poder de representatividade em sistemas de estacionamento público em vias urbanas; pois, pelas suas características de aleatoriedade, incorporam a variabilidade da demanda ao longo do tempo.

A técnica de Pesquisa Operacional (PO) utilizada nesse trabalho é a Teoria de Filas, que permite encontrar, através de análises matemáticas detalhadas, um ponto de equilíbrio que satisfaça o cliente e seja viável economicamente para o provedor do serviço.

$\mathrm{Na}$ maioria dos casos, os três processos básicos do método da Teoria de Filas (processo de chegadas, processo de atendimento e dimensionamento) fornecem as etapas adequadas para o estudo de um sistema de estacionamento.

\section{Processo de Chegadas}

O processo de chegadas de clientes em um sistema é representado pela Equação 1:

$\lambda=\frac{\mathrm{N}}{\mathrm{T}}$

Onde:

$\lambda$ - taxa de chegadas (veíc/min);

$\mathrm{N}$ - número de chegadas (veíc); e

$\mathrm{T}$ - tempo de observação (min). 
O índice de congestionamento de um sistema representa a taxa de utilização do serviço. Se o valor do índice de congestionamento for menor que 1, significa que não há formação de filas. Se for maior que 1, significa que há formação de filas. Se for igual a 1, significa que não há formação de filas, mas o sistema está operando à máxima capacidade, ou seja, todos os canais de atendimento estão sempre ocupados.

\section{RESULTADOS E DISCUSSÃO}

\section{Segmento Norte-Sul (Zona Norte)}

A taxa de chegada de veículos de carga e descarga para estacionar é calculada pela Equação 1, utilizando o número de veículos que chegam na hora de pico (15 veículos, entre 10 e 11h). Assim, a taxa de chegada $(\lambda)$ é igual a 0,25 veículos por minuto.

O tempo médio de estacionamento é igual a 27 minutos por veículo de carga. Assim, a taxa de atendimento $(\mu)$ do estacionamento para os veículos de carga, calculada pela Equação 2 resulta em 0,04 minutos por veículo.

O número de canais de atendimento $(\mathrm{C})$ para o estacionamento dos veículos de carga, calculado pela Equação 3, é igual a 8 baias, considerando o Índice de Congestionamento $(\rho)$ igual a 0,8 .

Após o cálculo do número de vagas do estacionamento, realizou-se uma simulação com os dados reais do centro comercial estudado para verificar se a quantidade de vagas calculada é satisfatória à demanda dos veículos de carga e descarga. A simulação demonstrou que uma área de estacionamento com 8 vagas seria suficiente para atender a demanda.

Portanto, a área dimensionada com 8 baias é caracterizada por um bolsão de estacionamento para os veículos de carga e descarga, constituída da seguinte forma: 2 baias para caminhões pequenos, 4 baias para caminhões médios e 2 baias para caminhões grandes, seguindo a mesma proporção entre veículos pequenos, médios e grandes obtida na coleta de dados. 


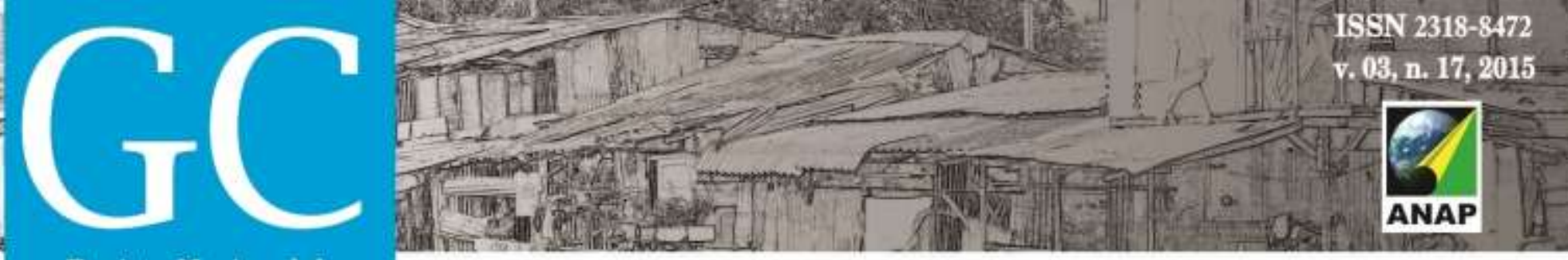

Revista Nacional de

Gerenciamento de Cidades

A Figura 1 mostra o bolsão de estacionamento dimensionado para a utilização específica dos veículos de carga e descarga que realizam as atividades na área em estudo.

Figura 1: Bolsão de estacionamento para veículos de carga e descarga no segmento Norte-Sul (Zona Norte)

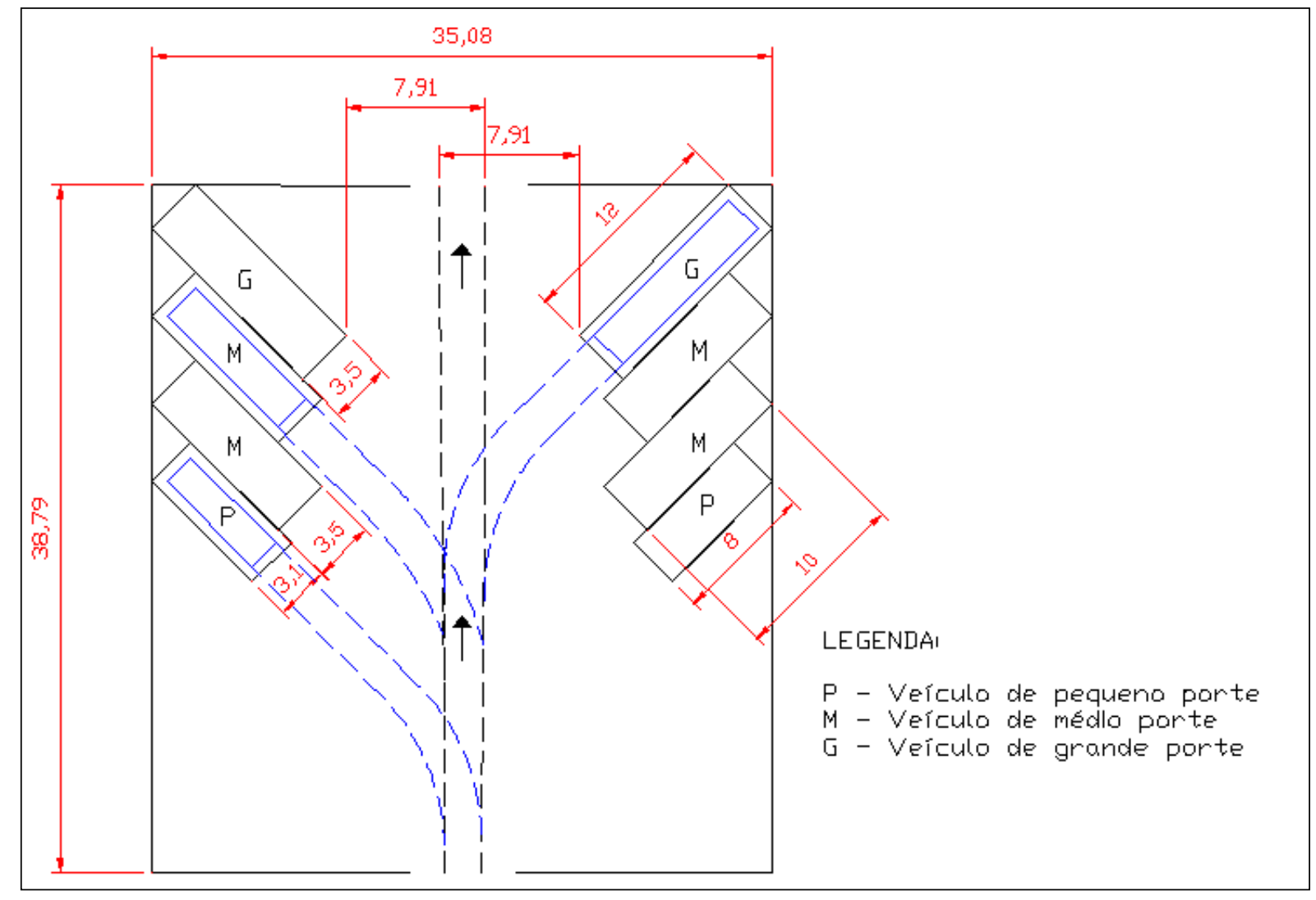

Segmento Sul-Norte (Zona Norte)

Os cálculos das Equações 1, 2 e 3 resultaram em: taxa de chegada $(\lambda)$ igual a 0,184 veículos por minuto (para 11 veículos na hora de pico, entre 9 e 10h); taxa de atendimento $(\mu$ ) igual 0,059 minutos por veículo (com tempo médio de estacionamento igual a 17 minutos por veículo de carga); e número de canais de atendimento $(C)$ igual a 4 baias (com índice de congestionamento igual a 0,8 ). 


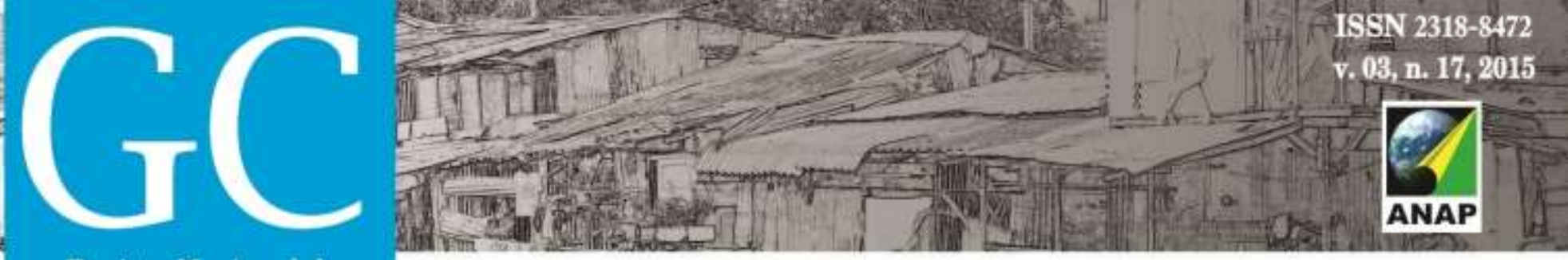

Revista Nacional de

Gerenciamento de Cidades

Comparando o número de canais de atendimento (4 baias) com a simulação com os dados reais do centro comercial estudado, verificou-se que a quantidade de vagas calculada é insatisfatória à demanda dos veículos de carga e descarga, havendo a formação de filas. Portanto, a quantidade de vagas para veículos de carga suficiente para atender a demanda é igual a 6, valor obtido na simulação para que não ocorra a formação de filas.

A Figura 2 mostra o bolsão de estacionamento dimensionado para a utilização específica dos veículos de carga e descarga que realizam as atividades na área em estudo.

Figura 2: Bolsão de estacionamento para veículos de carga e descarga no segmento Sul-Norte (Zona Norte)

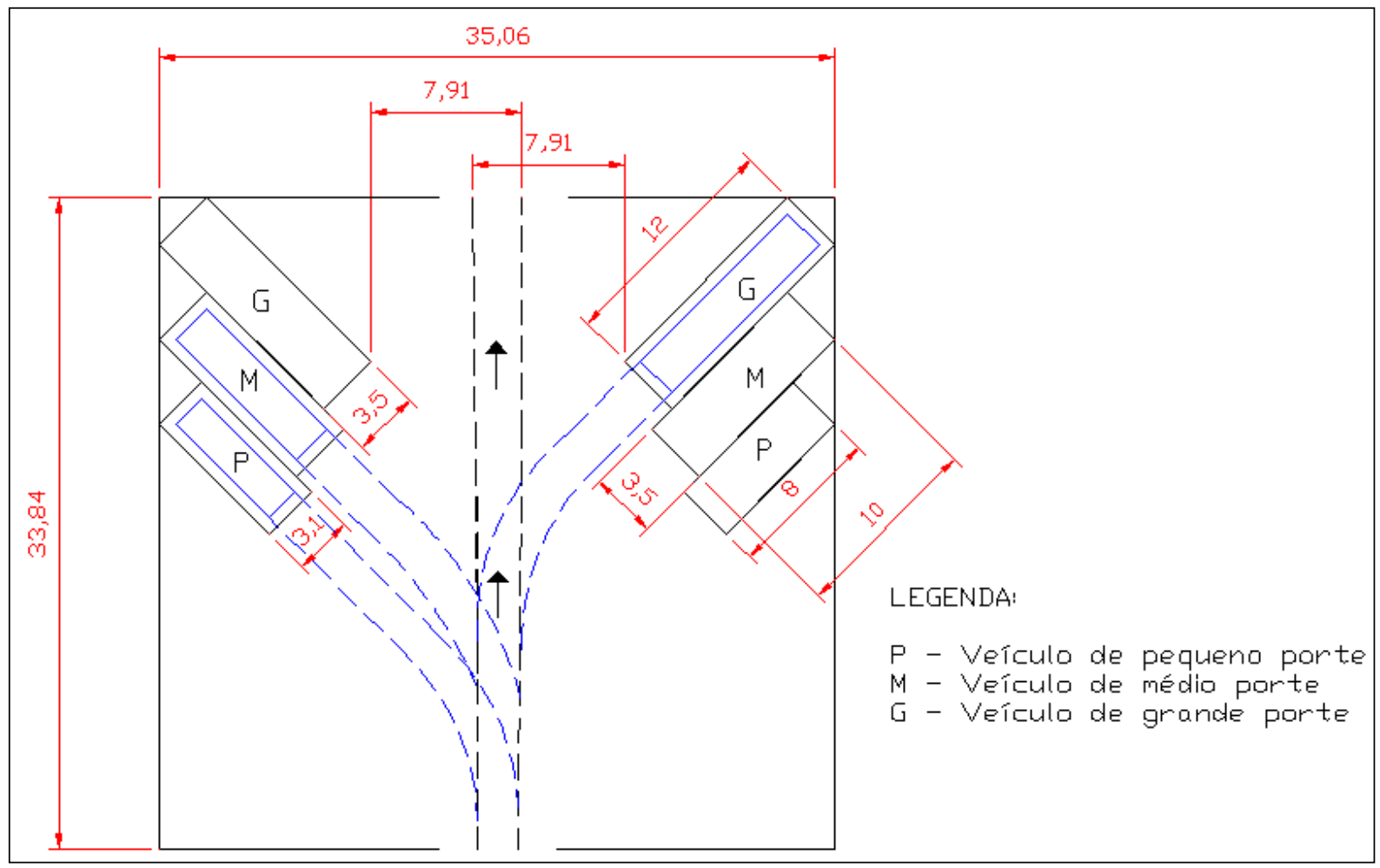




\section{CONCLUSÕES}

O desenvolvimento deste trabalho demonstrou que o centro comercial em estudo necessita de duas áreas específicas para estacionamento de carga e descarga de veículos: uma com 8 vagas para o segmento Norte-Sul e outra com 6 vagas para o segmento Sul-Norte.

Ao longo da principal via de comércio da cidade não há nenhuma vaga demarcada para operações de carga/descarga. Os veículos de mercadorias que chegam para estacionar são de porte pequeno, médio e grande.

O caminhão de pequeno porte ocupa o equivalente a um automóvel e meio no estacionamento ao longo da via. O caminhão de porte médio ocupa uma área igual a duas vagas de estacionamento para automóvel. O caminhão de grande porte ocupa o equivalente a cinco automóveis no estacionamento ao longo da via.

Uma área específica para o estacionamento dos veículos de carga e descarga é muito importante para garantir a segurança da população e permitir a fluidez do tráfego, além de reduzir a emissão de poluentes na cidade.

Diversos problemas no centro comercial em estudo seriam solucionados com o estacionamento específico para carga e descarga. Os principais problemas detectados se constituem na ação de estacionamento proibido nos seguintes locais: vaga específica de farmácia, garagem, faixa de pedestre, faixa amarela, rotatória, vaga para deficiente, vagas para motocicleta, formação de fila dupla e guia rebaixada.

Portanto, pode-se concluir que a compatibilidade de todos os agentes envolvidos no transporte urbano de carga deve ser inserida ao sistema urbano como um todo, caracterizado pela integração entre as partes e não pelo desempenho dos seus componentes separadamente. 
